\title{
An Efficient, and Fast Convergent Algorithm for Barrier Options
}

\author{
Tian-Shyr Dai ${ }^{1}$ and Yuh-Dauh Lyuu ${ }^{2}$ \\ 1. Department of Information and Finance Management, National Chiao-Tung University, 1001 Ta \\ Hsueh Road, Hsinchu, Taiwan 300, ROC. E-mail:d88006@csie.ntu.edu.tw. He was supported by \\ NSC grant 94-2213-E-033-024. \\ 2. Department of Computer Science \& Information Engineering, National Taiwan University.
}

\begin{abstract}
A barrier option is an option whose payoff depends on whether the price path of the underlying asset ever reaches certain predetermined price levels called the barriers. A single- (double-) barrier option is a barrier option with one (two, respectively) barrier(s). No simple and exact closed-form pricing formula for double-barrier options has been reported in the literature. This paper proposes a novel tree model that can price both single- and double-barrier options efficiently and accurately. This tree model achieves the high efficiency by combinatorial techniques and numerical accuracy by hitting the barriers exactly. Numerical experiments are given to verify the superiority of our method.
\end{abstract}

Keywords: barrier option, combinatorics, option pricing, tree

\section{Introduction}

A barrier option is an option whose payoff depends on whether the stock price reaches a certain predetermined level (the barrier) before the maturity date. A double- and the single-barrier options are barrier options with two and one barriers, respectively. A knock-in barrier option comes into existence if the stock price reaches the barrier(s) before the maturity date, while a knock-out one ceases to exist if the stock price reaches the barrier(s) before the maturity.

When the payoff functions for the single barrier options follow some standard forms, analytical pricing formulas are derived in [8]. The valuation of doublebarrier options is discussed in $[5,11,6]$. However, there are no simple, exact closed-form formulas for these options. The pricing formula can be expressed as an infinite series of cumulative normal distributions. Although truncation of this series is necessary numerically, it can lead to large pricing errors [6].

A tree model is popular for pricing barrier options. This research is interesting since it can price barrier options with nonstandard payoff functions, such as power payoff functions. However, there may not be closed-form formulas for such options. A tree divides the time span from now to the option's maturity into $n$ time steps and simulates the stock price discretely at each step. Take the 3-time-step CRR tree [2] illustrated in Fig. 1 as an example. Let the stock price at time step 0 be $S_{0}$. From an arbitrary node with price $S^{\prime}$, the CRR tree says that the stock price after one time step equals $S^{\prime} u$ (the up move) with 


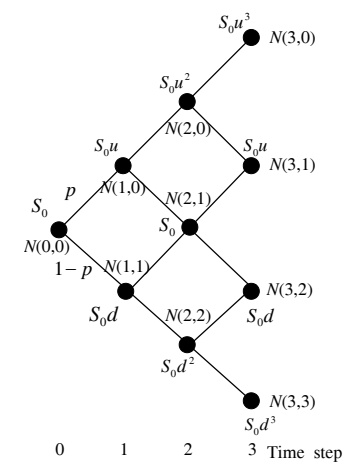

Fig. 1. The CRR Tree.

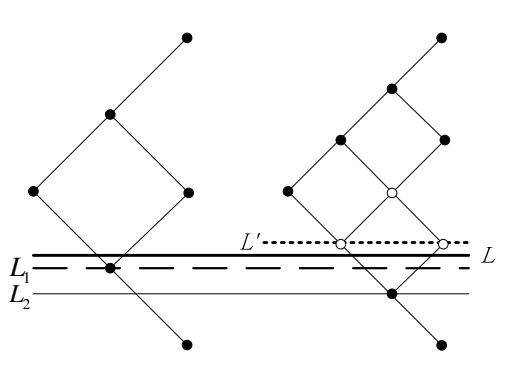

(a) (b)

Fig. 2. Pricing a Single-Barrier Option by the CRR Tree Model. The barrier is denoted by $L$. (a) The effective barrier is $L_{1}$. (b) The effective barrier is $L_{2}$.

probability $p$ and $S^{\prime} d$ (the down move) with probability $1-p$, where $u d=1$. Hence the price resulting from $j$ down moves and $i-j$ up moves from time step 0 equals $S_{0} u^{i-j} d^{j}$ with probability $\left(\begin{array}{l}i \\ j\end{array}\right) p^{i-j}(1-p)^{j}$. This node at time step $i$ is denoted as $N(i, j)$ for simplicity. Some details will be introduced in Section 2.

Pricing barrier options on a CRR tree makes the computed prices oscillate significantly as a function of $n$ [1]. This is because the barrier being assumed by the tree varies with $n$. Consider the pricing of a single-barrier option with barrier $L$ in Fig. 2. In the 2-time-step tree of panel (a), the stock price can not hit the barrier $L$ exactly. Instead, a near price on the CRR tree like $L_{1}$ becomes the barrier adopted. Call it the effective barrier. Similarly, in the 3-time-step tree of panel (b), the effective barrier is changed to $L_{2}$. The pricing results oscillate mainly because the effective barrier fluctuates with $n$. To solve this problem, Boyle and Lau suggest to pick a proper $n$ for which the tree has a layer close to $L$ [1]. This method reduces the errors dramatically. However, their method can not be easily adapted to handle two barriers since it is next to impossible to pick an $n$ that will tailor to both barriers. Derman et al. price the single-barrier options by interpolation [3]. They first calculate the option value for each hallow node by moving the barrier outward to $L_{2}$. Then they calculate the value for 
each hallow node by moving the barrier inward to $L^{\prime}$. We call $L^{\prime}$ and $L_{2}$ the inner and outer barriers to $L$, respectively. The option value for each hollow node is obtained by interpolating the two values mentioned above.

Ritchken alleviate the oscillation problem [10] by using "stretch parameter(s)" to tune the structure of his trinomial tree. Thus a layer of his tree can be made to coincide with each barrier. However, the branching probabilities of Ritchken's trinomial tree model are not guaranteed to be valid. More seriously, when a barrier is very close to the initial stock price, a large number of time steps is required and his algorithm becomes inefficient. Figlewski and Gao suggest the adaptive mesh model (AMM) to solve this "barrier-too-close" problem for pricing single-barrier options [4]. But no efforts have been carried out to extend the AMM to price double-barrier options.

All aforementioned approaches are not efficient enough since each node of the tree must be evaluated during backward induction to obtain the price. As the number of nodes of a tree is proportional to $n^{2}$, the backward induction also runs in $O\left(n^{2}\right)$ time. Lyuu provides an $O(n)$-time combinatorial algorithm for pricing single-barrier options on the CRR tree [7]. However, the pricing results oscillate significantly since the CRR tree is not guaranteed to hit the barrier.

This paper proposes a novel tree model, the tri-binomial tree (the TB tree hereafter), for pricing barrier options. The TB tree draws mainly from $[7,10]$. First, to alleviate the oscillation problem, the TB tree is guaranteed to have a layer that coincides with each barrier. Second, the TB tree is built on the CRR tree and the computation can be made linear in $n$ by combinatorial techniques. We also prove that TB tree is constructible and valid. Numerical results show that our approach can achieve the same level of accuracy with much less computational time than other tree approaches mentioned above.

\section{Preliminaries}

We assume that the option initiates at time 0 and matures at time $T$. The exercise price for this option is denoted by $X$. Let $S_{t}$ denote the stock price at time $t$, where $0 \leq t \leq T$. $S_{t}$ follows the log-normal diffusion process:

$$
S_{t+d t}=S_{t} \cdot \exp \left[\left(r-0.5 \sigma^{2}\right) d t+\sigma d W_{t}\right],
$$

where $W_{t}$ is the standard Wiener process, $r$ is the risk-free interest rate per annum, and $\sigma$ denotes the volatility of the stock price.

A tree model divides the time span from time 0 to time $T$, into $n$ time steps and simulates the price discretely at each time step. A tree converges to the stock price process mentioned in Eq. (1) if the first and the second moments of the stock price process are matched at each node of the tree. Consider the CRR tree illustrated in Fig. 1. To match the first two moments of the stock price, $u$ is set as $e^{\sigma \sqrt{T / n}}$. The probability $p$ is set to $\left(e^{r T / n}-d\right) /(u-d)$.

The payoffs of the barrier options at time $T$ are defined as follows. Define $S_{\text {inf }}=\inf _{0 \leq t \leq T} S_{t}$. The payoff of a down-and-out single-barrier call option with barrier $L$ is

$$
\text { Payoff }= \begin{cases}0, & \text { if } S_{\text {inf }} \leq L, \\ \max \left(S_{T}-X, 0\right), & \text { otherwise }\end{cases}
$$




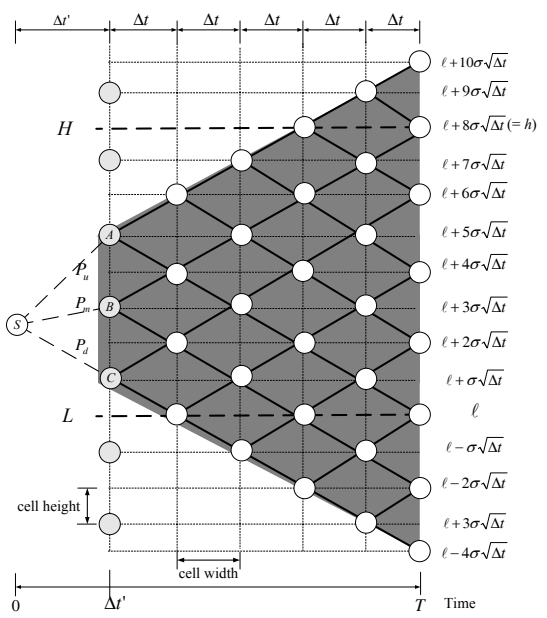

Fig. 3. The TB tree for Pricing Double-Barrier Options. $L$ and $H$ are denoted by thick dotted lines. The $S_{0}$-log-prices for the nodes at maturity are listed next to these nodes.

Define $S_{\text {sup }}=\sup _{0 \leq t<T} S_{t}$. The payoff of a knock-in double-barrier call option with barriers $L$ and $H$ is

$$
\text { Payoff }= \begin{cases}\max \left(S_{T}-X, 0\right), & \text { if } S_{\mathrm{sup}} \geq H \text { or } S_{\mathrm{inf}} \leq L, \\ 0, & \text { otherwise. }\end{cases}
$$

This paper focuses on the pricing aforementioned barrier options. The extension to other types of barrier options is straightforward.

\section{Construction of the TB Tree}

The TB tree is built on the CRR tree and is guaranteed to hit each barrier. We will first show how to construct an TB tree. The $O(n)$-time combinatorial algorithm for pricing double-barrier options will be introduced in section 4 .

Consider the TB tree for a double-barrier option with barriers $H$ and $L$ in Fig. 3. The underlying is a CRR tree in shadow. The first two time steps of the CRR tree is truncated, and this CRR tree begins with three nodes: $A, B$, and $C$. These three nodes are connected by $S$ at time 0 . The TB tree has two following features: (1) It has two layers that coincide with $H$ and $L$. (2) The branching probabilities $P_{u}, P_{m}$, and $P_{d}$ are valid. To make the truncated CRR tree hit $H$ and $L$, the length of a time step $\Delta t$ should satisfy some specific constraint. We will explain how to pick a proper $\Delta t$ later. The length of the the first time step of the TB tree $\Delta t^{\prime}$ is the remaining amount of time to make the whole tree span $T$. $A, B$, and $C$ are finally selected among the light gray nodes at time $\Delta t^{\prime}$ to make $P_{u}, P_{m}$, and $P_{d}$ valid and to make the TB tree hit $H$ and $L$.

Now we determine $\Delta t$. Assume that the stock price at $S$ is $S_{0}$. Define the $S_{0^{-}}$ $\log$-price of price $V^{\prime}$ as $\ln \left(V^{\prime} / S_{0}\right)$ for convenience. A $S_{0}$-log-price of $z$ therefore 
implies a price of $S_{0} e^{z}$. Note that the difference between the $S_{0}$-log-prices of two adjacent nodes like $A$ and $B$ is $2 \sigma \sqrt{\Delta t}$, because the upward and the downward multiplication factors of the CRR tree are $e^{\sigma \sqrt{\Delta t}}$ and $e^{-\sigma \sqrt{\Delta t}}$, respectively. The $S_{0}$-log-prices of $H$ and $L$ are $h=\ln \left(H / S_{0}\right)$ and $\ell=\ln \left(L / S_{0}\right)$, respectively. To make sure the truncated CRR tree has two layers that coincide with $H$ and $L$, $\frac{h-\ell}{2 \sigma \sqrt{\Delta t}}$ must be some integer $k$. Assume that we try to construct an $m$-time-step tree. The length of each time step $\Delta \tau=T / m$, but $\frac{h-\ell}{2 \sigma \sqrt{\Delta \tau}}$ may not be an integer. So we pick a $\Delta t$ that is close to $\Delta \tau$ and that makes $\frac{h-\ell}{2 \sigma \sqrt{\Delta t}}$ an integer. We pick $\Delta t=\left(\frac{h-\ell}{2 \kappa \sigma}\right)^{2}$, where $\kappa=\left\lceil\frac{h-\ell}{2 \sigma \sqrt{\Delta \tau}}\right\rceil$. Note that the number of time steps of TB tree is no longer equal to, but close to, $m$ as we change the length of each time step. Let the truncated CRR tree has $\left\lfloor\frac{T}{\Delta t}\right\rfloor-1$ time steps. The length of the first time step $\Delta t^{\prime}$ is the remaining amount of time to make the whole tree span $T: \Delta t^{\prime}=T-\left(\left\lfloor\frac{T}{\Delta t}\right\rfloor-1\right) \Delta t$. It is easy to verify that $\Delta t \leq \Delta t^{\prime}<2 \Delta t$.

Finally, we select $A, B$, and $C$ that are connected by $S$. Note that three branches are required for $S$ to match the first two moments of the logarithmic stock price process; a binomial branch from $S$ does not have enough degrees of freedom. By Eq. (1), the mean $\mu$ and the variance Var of the $S_{0}$-log-prices of $A$, $B$, and $C$ equal $\left(r-\sigma^{2} / 2\right) \Delta t^{\prime}$ and $\sigma^{2} \Delta t^{\prime}$, respectively. Let the $S_{0}$-log-price of $B$ be $\hat{u}$. To make the truncated CRR tree hit $H$ and $L$, the following must be satisfied for some integer $\mathrm{j}$ :

$$
\hat{u}= \begin{cases}\ell+2 j \sigma \sqrt{\Delta t}, & \text { if the truncated CRR tree has even number of time steps }, \\ \ell+(2 j+1) \sigma \sqrt{\Delta t}, & \text { otherwise. }\end{cases}
$$

Those nodes whose $S_{0}$-log-prices satisfy the above constraint at time $\Delta t^{\prime}$ are colored in light gray in Fig. 3. We choose $A, B$, and $C$ to make $P_{u}, P_{m}$, and $P_{d}$ valid. Recall that the difference between two adjacent nodes' $S_{0}$-log-prices is $2 \sigma \sqrt{\Delta t}$. There exists a unique light gray node whose $S_{0}$-log-price lies in the interval $[\mu-\sigma \sqrt{\Delta t}, \mu+\sigma \sqrt{\Delta t})$. We select this node as $B$. For example, the $S_{0^{-}}$ $\log$-price of $B$ is $\ell+3 \sigma \sqrt{\Delta t}$ in Fig. 3. The $S_{0}$-log-price of $B$ is closest to $\mu$ among those of the light gray nodes. The $S_{0}$-log-prices of $A$ and $C$ are $\hat{\mu}+2 \sigma \sqrt{\Delta t}$ and $\hat{\mu}-2 \sigma \sqrt{\Delta t}$, respectively. Define $\beta \equiv \hat{\mu}-\mu, \alpha \equiv \beta+2 \sigma \sqrt{\Delta t}, \gamma \equiv \beta-2 \sigma \sqrt{\Delta t}$. Note that $\beta \in[-\sigma \sqrt{\Delta t}, \sigma \sqrt{\Delta t})$ and that $\alpha>\beta>\gamma$. The branching probabilities are derived by solving the following equalities

$$
\begin{aligned}
P_{u} \alpha+P_{m} \beta+P_{d} \gamma & =0, \\
P_{u} \alpha^{2}+P_{m} \beta^{2}+P_{d} \gamma^{2} & =\text { Var, } \\
P_{u}+P_{m}+P_{d} & =1 .
\end{aligned}
$$

Eqs. (2) and (3) match the first two moments of the logarithmic stock price, and Eq. (4) ensures that $P_{u}, P_{m}, P_{d}$ as probabilities sum to one. A proof given in Appendix A shows that the inequalities $0 \leq P_{u}, P_{m}, P_{d} \leq 1$ are satisfied.

We now develop an efficient and accurate algorithm for pricing double-barrier options on the TB tree. First, an $O(n)$-time algorithm is developed to price a double-barrier option on the CRR tree in section 4 . This algorithm can be used to evaluate the option values of $A, B$, and $C$ efficiently since each of these nodes can be viewed as the root node of a CRR tree that begins at time $\Delta t^{\prime}$. The final 
pricing result of our TB tree is $e^{-r \Delta t^{\prime}}\left(P_{u} \times V_{A}+P_{m} \times V_{B}+P_{d} \times V_{C}\right)$, where $V_{X}$ denotes the option value at $X$.

Pricing a single-barrier option with barrier $L$ by the TB tree is much simpler! We simply construct the truncated CRR tree that has a layer coinciding with $L$. Note that we do not need to adjust the length of a time step since it needs to match only $L$ (instead of $L$ and $H$ ).

\section{An $O(n)$-Time Combinatorial Algorithm on a CRR Tree}

We first derive a useful combinatorial formula by the reflection principle and the inclusion-exclusion principle. This formula is used to build up the pricing algorithm. We focus on the knock-in double-barrier call options. The extension to other types of double-barrier options is straight forward.

\section{A Combinatorial Formula}

A combinatorial formula is derived with the help of the lattice in Fig. 4. This lattice reflects the structure of the CRR tree. The $x$ - and $y$-coordinates denotes the time step of the tree and the stock price level, respectively. To fit the price movement on the CRR tree, each path can move from vertex $(i, j)$ to vertex $(i+1, j+1)$ (the up move) or vertex $(i+1, j-1)$ (the down move). Now consider the following problem: How many price paths starting form $A$ will reach either $H$ or $L$ before arriving at $B$ ? Without loss of generality, we assume that $a, b \geq 0$. A simplified problem is discussed first: How many paths moving

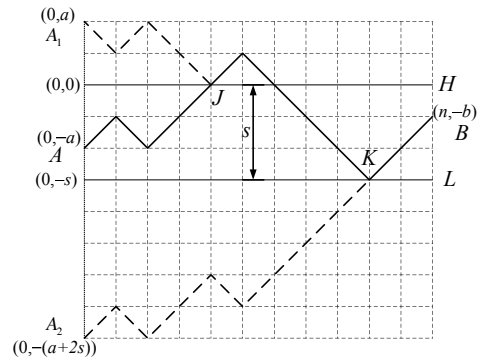

Fig. 4. Count the Number of Paths That Hit Barrier $H$ and $L$ by the Reflection Principle.

form $A$ to $B$ will hit barrier $H$ before one hit of barrier $L$ ? One such path may hit barrier $H$ at $J$ and barrier $L$ at $K$. We can reflect the path $\widehat{A J}$ (marked by solid curve) with respect to the $H$-axis to get $\widehat{A_{1} J}$ (marked by dash curve). Each path from $A$ to $J$ maps to a unique path from $A_{1}$ to $J$. Thus the number of paths from $A$ to $J$ equals to the number of paths from $A_{1}$ to $J$. The reflection principle says that the number of paths staring from $A$ and hitting $H$ before reaching $B$ equals to the number of paths moving from $A_{1}$ to $B$. The reflection principle can be applied more than once. The curve $\widehat{A_{1} K}$ can be reflected with respect to the $L$-axis to obtain $\widehat{A_{2} K}$. Again, the number of paths staring from 
$A_{1}$ and hitting $L$ before reaching $B$ equals to the number of paths moving from $A_{2}$ to $B$. Thus the number of paths moving from $A$ to $B$ and reaching $H$ at least once before reaching $L$ is equal to the number of paths moving from $A_{2}$ to $B$. Assume that $x$ up moves and $y$ down moves are required to move from $A_{2}$ to $B$. Thus we have $x+y=n$ and $x-y=a-b+2 s$. We get $x=\frac{n+a-b+2 s}{2}$ by solving the above equations. So the answer to the aforementioned problem is

$$
\left(\begin{array}{c}
n \\
\frac{n+a-b+2 s}{2}
\end{array}\right) \text { for even, non-negative } n+a-b .
$$

Note that a path counted by Eq. (5) may hit $L$ first before hitting $H$. The point is that among the hits, one hit of $H$ must appear before one hit of $L$.

The problem of counting the number of paths that will hit either $H$ or $L$ before arriving at $B$ is now within reach. First, a function $f$ is constructed to map each path to a string. This string contains the information about the barrier hitting sequence. For example, $f(\widehat{A B})=H H L$ since $\widehat{A B}$ hits $H$ twice before hitting $L$. Next, we define $\alpha_{i}$ as the set of paths whose $f$ value contains $\overbrace{H^{+} L^{+} H^{+}}^{i}$

$\overbrace{H^{+} L^{+} H^{+} \ldots} L^{+}$and $H^{+}$denote a sequence of $L$ s and $H$ s, respectively. Obviously, $\widehat{A B}$ belongs to set $\alpha_{1}$ and set $\alpha_{2}$. Similarly, define $\beta_{i}$ as the set of paths whose $f$ value contains $\overbrace{L^{+} H^{+} L^{+} \ldots}^{i}$. Thus the path $\widehat{A B}$ belongs to set $\beta_{1}$. The number of elements in set $\alpha_{i}$ and $\beta_{i}$ can be calculated by repeatedly using the reflection principle. The number of elements in each set is listed as follows:

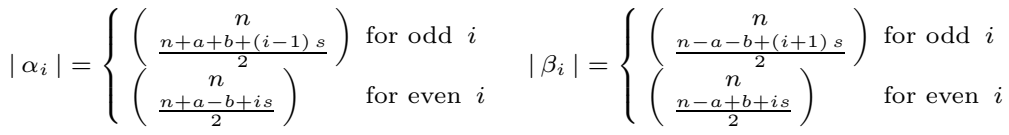

Note that each path that hits the barrier may belong to more than one set. For example, $\widehat{A B}$ belongs to $\alpha_{1}, \alpha_{2}$, and $\beta_{1}$. The inclusion-exclusion principle is then applied to calculate the number of paths that moves from $A$ (with coordinate $(0,-a))$ to $B(n,-b)$ and that hit either $H$ or $L$ at least once as follows:

$$
\mathbf{N}(a, b, s)=\sum_{i=1}^{\left\lceil\frac{n}{s}\right\rceil}(-1)^{i+1}\left(\left|\alpha_{i}\right|+\left|\beta_{i}\right|\right) .
$$

\section{The Pricing Algorithm}

The construction of the pricing algorithm can be divided into several different cases. For some degenerate cases like $S_{0} \leq L, S_{0} \geq H$, and $X \geq H$, the value of a knock-in double-barrier option can be proved to equal the value of a vanilla call option. These degenerate cases can be directly priced by any analytical or numerical method that price a vanilla call option. So we further focus on the case $L<S_{0}<H$ and $X<H$ from now on. The pricing result is obtained by summing the value contributed by each node at the maturity of the CRR tree. Combinatorial techniques is applied to implement our pricing algorithm efficiently. We first put the CRR tree on a lattice as displayed in Fig. 5. Assume that the barrier $H$ and $L$ equal to $S_{0} u^{n-h} d^{h}\left(=S_{0} u^{n-2 h}\right)$ and $L=S_{0} u^{n-l} d^{l}\left(=S_{0} u^{n-2 l}\right)$, respectively. The exercise price $X$ satisfies the following equality $S_{0} u^{n-a} d^{a} \leq X<S_{0} u^{n-a+1} d^{a-1}$ for some integer $a$. 


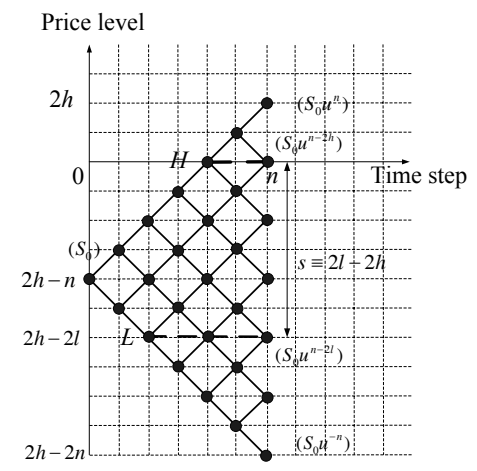

Fig. 5. Put a CRR Tree on a Lattice. The coordinate of the root node of the CRR tree is $(0,2 h-n)$. The values in parentheses denote the stock prices.

Now we analyze the value contributed by a price path that reaches $N(n, j)$. The probability for this path is $p^{n-j}(1-p)^{j}$. The payoff at $N(n, j)$ is $\left(S_{0} u^{n-j} d^{j}-\right.$ $X)^{+}$. Thus the value contributed by this path is $p(j) \equiv e^{-r T} p^{n-j}(1-p)^{j}\left(S_{0} u^{n-j} d^{j}-\right.$ $X)^{+}$, if this price path hits the barrier. Note that the value contribution by $N(n, j)$ is $V(j) \equiv\left(\begin{array}{l}n \\ j\end{array}\right) p(j)$ if $N(n, j)$ is above $H$ or below $L$ (inclusive). This is because all the price paths that reach $N(n, j)$ must also hit the barrier.

The pricing algorithm can be divided into two following cases. For convenience, the term "terminal node" refers to the node of the tree at maturity date. Case 1. $L<X<H$ : The option value can be decomposed into two parts: (1) the value contributed by the terminal nodes between $X$ and $H$, and (2) the value contributed by the terminal nodes above $H$ (inclusive). The first part can be computed by accumulating the values contributed by the terminal nodes, says $N(n, j)(a>j>h)$, between $X$ and $H$. The number of paths that reach one of the barriers before reaching $N(n, j)$ is $\mathbf{N}(n-2 h, 2 j-2 h, 2 l-2 h)$ (see Eq. (7)). The value contributed by such a path is $p(j)$. Therefore, the value contributed by node $N(n, j)$ is $\mathbf{N}(n-2 h, 2 j-2 h, 2 l-2 h) p(j)$. The first part of the option value is $\left.P_{0} \equiv \sum_{j=h+1}^{a-1} \mathbf{N}(n-2 h, 2 j-2 h, 2 l-2 h)\right) p(j)$. The second part of the option value is computed by accumulating the values contributed by the terminal nodes, says $N(n, i)(0 \leq i \leq h)$, above the barrier $H$ (inclusive). The value contributed by $N(n, i)$ is $V(i)$. Therefore, the second part of the option value is $P_{1}=\sum_{i=0}^{h} V(i)$. The value of a knock-in double-barrier call option is $P_{0}+P_{1}$.

Case 2. $X \leq L$ : The option value is decomposed into three parts: (1) the value contributed by the terminal nodes between $L$ and $H,(2)$ the value contributed by the terminal nodes above $H$ (inclusive), and (3) the value contributed by the terminal nodes between $L$ (inclusive) and $X$. The first part of the option value is expressed as $P_{0}^{\prime} \equiv \sum_{j=h+1}^{l-1} \mathbf{N}(n-2 h, 2 j-2 h, 2 l-2 h) p(j)$. The second part of the option value equals $P_{1}$. The third part is computed by accumulating the values contributed by the terminal nodes, says $N(n, k)(l \leq k<a)$, between $L$ (inclusive) and $X$ to get $P_{1}^{\prime} \equiv \sum_{k=l}^{a} V(k)$. The option value is $P_{0}^{\prime}+P_{1}+P_{1}^{\prime}$. 


\section{Experimental Results}

We first compare the performance among the TB tree, Lyuu's algorithm [7], and the Ritchken's trinomial tree [10] in Table 1. All programs are run on a Pentium-4 2.8GHz computer. Note that Lyuu's algorithm oscillates significantly. Both Ritchken's trinomial tree and the TB tree converge monotonically to the true value 5.9968. But the TB tree can achieve each level of accuracy with fewer computational time than the Ritchken's trinomial tree. Thus we conclude that the TB tree is superior to both Lyuu's and the Ritchken's approaches.

Table 1. Convergence Rate for Pricing a Down-and-Out Single-Barrier Option.

\begin{tabular}{|r||r|r||r|r||r|r|}
\hline \multirow{2}{*}{ Time (sec) } & \multicolumn{2}{c||}{ Ritchken } & \multicolumn{2}{c|}{ Lyuu } & \multicolumn{2}{|c|}{ TB } \\
\cline { 2 - 7 } & $n$ & Value & $n$ & Value & $n$ & Value \\
\hline 0.001 & 100 & 5.9997 & 1000 & 6.1002 & 500 & 5.9980 \\
\hline 0.004 & 200 & 5.9986 & 4000 & 6.1998 & 2000 & 5.9972 \\
\hline 0.016 & 400 & 5.9980 & 16000 & 6.0829 & 8000 & 5.9969 \\
\hline
\end{tabular}

The initial stock price is 95 , the exercise price is 100 , the risk-free rate is $10 \%$ per annum, the volatility of the stock price is $25 \%$, the time to maturity is 1 year, and the barrier is 90 . Time denotes the computational time in seconds. $n$ and Value denotes the number of time steps and the pricing result of each tree model.

The TB tree can accurately solve the barrier-too-close problem for pricing double-barrier options while AMM can not as illustrated in Table 2. Level denotes the AMM level. The number of steps of AMM is determined by the AMM level. The Inner Barrier and the Outer Barrier denote the results computed by moving the upper barrier (120) to the inner barrier and the outer barrier, respectively. Note that AMM undervalues the option if the upper barrier moves down to the inner barrier and overvalues the option if the upper barrier moves up to the upper barrier. Each pricing result of the TB tree is properly selected so the number of steps of the TB tree is approximately equal to that of the AMM. Obviously, the TB tree provides more accurate results than the AMM.

\section{Conclusion}

The TB tree that can efficiently and accurately price barrier options is proposed in this paper. It is mainly composed of the CRR tree so the computation can be speeded up by combinatorial techniques. It produces accurate pricing results since it has a layer to coincide with each barrier. Numerical results show that the TB tree are superior to other existing approaches.

\section{References}

1. Boyle P., And LaU, S. "Bumping Against the Barrier with the Binomial Method," Journal of Derivatives, 1 (1994), pp. 6-14. 
Table 2. Convergence Rate Comparison between AMM and the TB tree.

\begin{tabular}{|r|r|r|r|r|r|}
\hline \multicolumn{5}{|c|}{ AMM } & \multicolumn{2}{|c|}{ TB } \\
\hline Level & $n$ & Inner Barrier & Outer Barrier & $n$ & Value \\
\hline 2 & 671 & 0.000001 & 0.000015 & 655 & 0.000003 \\
\hline 1 & 2686 & 0.000001 & 0.000005 & 2625 & 0.000003 \\
\hline
\end{tabular}

The initial stock price and the exercise price is 100 , the risk-free rate is $10 \%$, the volatility of the stock price is $30 \%$, the time to maturity is 1 year, and the two barriers are 99.5 and 120 , respectively. The pricing results of the TB tree converge to 0.000003 .

2. Cox J., Ross, S., And Rubinstein, M. "Option Pricing: A Simplified Approach." Journal of Financial Economics, 7 (1979), pp. 229-264.

3. Derman, E., Kani, I., Ergener, D., And Bardhan, I. "Enhanced Numerical Methods for Options with Barriers," Working Paper, Goldman Sachs. (1995).

4. Figlewski, S., And GaO, B. "The Adaptive Mesh Model: A New Approach to Efficient Option Pricing." Journal of Financial Economics, 53 (1999), pp. 313351.

5. Geman H., And Yor, M. "Pricing and Hedging Double-Barrier Options: A Probabilistic Approach." Mathematical Finance, 6 (1996), pp. 365-378.

6. Luo, L. "Various Types of Double Barrier Options." Journal of Computational Finance, 4 (2001), pp. 125-138.

7. LyuU Y.D. "Very Fast Algorithms for Barrier Option Pricing and the Ballot Problem." Journal of Derivatives, 5 (1998), pp. 68-79.

8. Merton, R. "Theory of Rational Option Pricing." Bell Journal of Economics and Management, 4 (1973), pp. 141-183.

9. Reiner, E. And Rubinstein, M. "Breaking Down the Barriers." Risk, 4 (1991), pp. 28-35.

10. Ritchken, P. "On Pricing Barrier Options." Journal of Derivatives, 3 (1995), pp. 19-28.

11. Sidenius, J. "Double Barrier Options: Valuation by Path Counting." Journal of Computational Finance, 1 (1998), pp. 63-79.

\section{A Validity of Risk-Neutral Probabilities}

Define det $=(\beta-\alpha)(\gamma-\alpha)(\gamma-\beta), \operatorname{det}_{u}=(\beta \gamma+\operatorname{Var})(\gamma-\beta), \operatorname{det}_{m}=(\alpha \gamma+$ $\operatorname{Var})(\alpha-\gamma)$, and $\operatorname{det}_{d}=(\alpha \beta+\operatorname{Var})(\beta-\alpha)$. Then Cramer's rule applied to Eq. (2)-(4) gives $P_{u}=\operatorname{det}_{u} / \operatorname{det}, P_{m}=\operatorname{det}_{m} / \operatorname{det}$, and $P_{d}=\operatorname{det}_{d} / \operatorname{det}$. Note that det $\langle 0$ because $\alpha>\beta>\gamma$. To ensure that the branching probabilities are valid, it suffices to show that $P_{u}, P_{m}, P_{d} \geq 0$. As det $<0$, it is sufficient to show $\operatorname{det}_{u}, \operatorname{det}_{m}, \operatorname{det}_{d} \leq 0$ instead. Finally, as $\alpha>\beta>\gamma$, it suffices to show that $\beta \gamma+\operatorname{Var} \geq 0, \alpha \gamma+\operatorname{Var} \leq 0$, and $\alpha \beta+\operatorname{Var} \geq 0$ under the premise $\beta \in[-\sigma \sqrt{\Delta t}, \sigma \sqrt{\Delta t})$. Indeed,

$\beta \gamma+\operatorname{Var}=\beta^{2}-2 \beta \sigma \sqrt{\Delta t}+\sigma^{2} \Delta t^{\prime} \geq \beta^{2}-2 \beta \sigma \sqrt{\Delta t}+\sigma^{2} \Delta t=(\beta-\sigma \sqrt{\Delta t})^{2} \geq 0$,

$\alpha \gamma+\operatorname{Var}=\beta^{2}-4 \sigma^{2} \Delta t+\sigma^{2} \Delta t^{\prime} \leq \beta^{2}-4 \sigma^{2} \Delta t+2 \sigma^{2} \Delta t=\beta^{2}-2 \sigma^{2} \Delta t \leq 0$,

$\alpha \beta+\operatorname{Var}=\beta^{2}+2 \beta \sigma \sqrt{\Delta t}+\sigma^{2} \Delta t^{\prime} \geq \beta^{2}+2 \beta \sigma \sqrt{\Delta t}+\sigma^{2} \Delta t=(\beta+\sigma \sqrt{\Delta t})^{2} \geq 0$,

as desired. 